\title{
Gérondif « non-coréférentiel »
}

Jun-ya WATANABE, Université de Tsukuba, Japon

\section{Introduction $^{1}$}

Cet article porte sur le gérondif dont le sujet n'est pas coréférentiel avec celui de la proposition régissante (désormais « gérondif non-coréférentiel »), illustré par les exemples (1) et (2), mais qui est souvent qualifié d'anomalie grammaticale, puisque dans (1), ce n'est pas l'appétit qui mange, ni la soif qui boit, et que dans (2), le sujet du gérondif « en le retrouvant » n'est pas « il » de la proposition principale.

(1) L'appétit vient en mangeant, la soif s'en va en buvant. (Rabelais, Gargantua)

(2) Au Prado, on s'est séparés à l'entrée pour voir les salles chacun de son côté. En le retrouvant à la sortie dans les jardins, il était avec un homme, il s'était fait alpaguer par un pédophile. (Hervé Guibert, Fou de Vincent)

En général, les grammaires normatives conseillent la coréférence entre les sujets de deux propositions :

(3) «Il est souhaitable, notamment, que le participe ou le gérondif détachés, surtout en tête d'une phrase ou d'une proposition, aient comme support le sujet de cette phrase et de cette proposition [...]»(Grevisse et Goose 1306).

Cependant, dans l'usage réel, l'emploi du gérondif non-coréférentiel est loin d'être une rare exception et semble garder une certaine productivité, comme on peut l'entrevoir avec l'exemple (2). Aussi aimerions-nous savoir à quelle(s) condition(s) son occurrence devient (relativement) acceptable.

Ci-après, nous procéderons en trois étapes. Nous examinerons d'abord, dans la première section, les solutions précédemment proposées pour en signaler les défauts. Ensuite, dans la section 2, nous présenterons les principaux résultats de notre enquête dans le corpus «Corpatext 1.02 ». Enfin, dans la section 3, en dégageant les dénominateurs communs aux exemples pris en compte, nous avancerons que le gérondif non-coréférentiel traduit un mode de cognition que l'on appelle le « mode I », celui avec le point de vue intérieur à la réalité décrite dans la phrase. 


\section{Solutions antérieures}

\subsection{Observation au niveau discursif : Marie-José Reichler-Béguelin (1995)}

Reichler-Béguelin (§.5) dit que « l'impression d'anomalie associée à certaines configurations syntaxiques atypiques provient très souvent du fait qu'elles ont été décontextualisées, et qu'on veut pouvoir les interpréter grammaticalement dans le cadre étroit et artificiellement autonomisé de la «phrase »». Ainsi, la phrase suivante est «nettement anacoluthique » quand elle est présentée à l'état isolé :

(4) Par exemple, c'est en coupant très court les cheveux de Twiggy, qui a de grandes oreilles, que son visage est devenu inoubliable. (Reichler-Béguelin §.5)

(4') Parfois, au contraire, on s'appuie sur les défauts physiques d'une femme pour créer un personnage hors du commun. Par exemple, c'est en coupant très court les cheveux de Twiggy, qui a de grandes oreilles, que son visage est devenu inoubliable. (idem)

Nous sommes tout à fait d'accord pour reconnaître l'importance du cotexte, mais ce n'est pas suffisant pour expliquer comment et pourquoi on peut employer le gérondif noncoréférentiel. Il faudrait montrer plus concrètement quel(s) élément(s) du contexte permet(tent) l'emploi de celui-ci.

\subsection{Rapport aux types d'emploi : Odile Halmoy (2003)}

Halmøy affirme que le gérondif non-coréférentiel n'est attestable que dans les quatre types d'emploi suivants :

(i) gérondif grammaticalisé :

(5) En partant de l'autoroute A1, prendre le périphérique au niveau de la porte de la Chapelle et allez jusqu'à la porte de Bercy. (115)

(ii) gérondif adverbial de phrase :

(6) En y pensant, le concept même d'une ville tenait du délire. (117)

(iii) gérondif marquant le repère temporel :

(7) En sortant du restaurant, les trottoirs étaient noirs et luisants, parsemés de givre et de neige fondue. (119)

(iv) la configuration A selon le terme de Halmøy (temps + cause, hypothèse, moyen) :

(8) En mettant la lettre à la poste ce soir avant huit heures, elle arrivera demain. (120)

Dans les autres types d'emploi, le sujet du gérondif est toujours coréférentiel avec celui du sujet.

(i) la configuration A' de Halmøy (relation d'inclusion ou d'équivalence) :

(9) En tuant sa mère, en étranglant le sordide, il a aussi assassiné le rêve. (100)

(ii) la configuration $\mathrm{B}$ de Halmøy (relation de concomitance (circonstance 
d'accompagnement)) :

(10) Je chante en me rasant. $\approx$ Je me rase en chantant. (102)

(iii) la configuration B' de Halmøy (relation d'hyponymie (manière)) $)^{2}$ :

(11) Elle répondit en bafouillant que c'était la fille d'une ancienne voisine. (104)

L'étude de Halmøy offre une précieuse description, mais elle n'explique pas pourquoi tels types d'emploi admettent la non-coréférence. Quel rapport, par exemple, existe-t-il entre le repère temporel et le gérondif non-coréférentiel ?

\subsection{Considérations théoriques en grammaire relationnelle : Géraldine Legendre (1989)}

Legendre avance une hypothèse selon laquelle « seul le syntagme nominal sur l'arc 1 peut contrôler le syntagme en en [=syntagme gérondif] » (779, ma traduction). C'est-à-dire que le sujet du gérondif doit occuper la position du sujet, notée « 1 », au moins sur une des diverses strates que suppose la grammaire relationnelle. Cette hypothèse a l'avantage de pouvoir récupérer les cas où le sujet du gérondif correspond à l'agent implicite derrière le passif d'une part (12), et au datif d'expérienceur de l'autre (13), car ces deux arguments constituent le sujet sur la strate de départ (c'est-à-dire le niveau le plus profond) dans cette théorie.

(12) De nouvelles victimes ont été découvertes en creusant dans le jardin. (ad loc.)

(13) Cette idée m'est venue en dormant. (780)

Mais la portée de cette hypothèse nous semble trop étroite, dans la mesure où elle ne s'intéresse qu'aux phénomènes privilégiés dans la grammaire relationnelle comme le datif d'expérienceur ou le passif. En effet, comment expliquer dans ce cadre les cas où aucun élément de la proposition régissante ne constitue le support du gérondif, comme dans l'exemple (4') ?

\subsection{Solutions en termes de " saillance " ou de "thématicité" : Martin Haspelmath (1995) et Yoshiyuki Kinouchi (2005)}

Haspelmath observe que le participant saillant dans le discours antérieur peut être le contrôleur du gérondif, comme dans :

(14) Il pensa une seconde que c'était sans doute cela qui l'avait sauvé, lui, trois mois plus tôt, mais en même temps, il cherchait un moyen de lui prouver le contraire. En y réfléchissant, c'était elle qui dès le début de leur liaison avait pris toutes les initiatives (35).

Et voici l'hypothèse de l'auteur :

(15) «Comme le contrôle se fait par un participant extrêmement saillant, et que le sujet est le plus souvent le participant le plus saillant de la proposition, l'assertion la plus économique serait que le sujet implicite est contrôlé par le participant le plus saillant» (36, ma 
traduction).

Nous pourrions formuler une critique contre Haspelmath : quand le sujet du gérondif n'apparaît nulle part textuellement, comme dans la phrase suivante, en quoi peut-on dire qu'il est le « participant le plus saillant»?

(16) Les peintures étaient en grande partie détruites. Le tableau de gauche en descendant l'escalier n'existait plus [...] (L. Dimier, Le Primatice, peintre, sculpteur et architecte des rois de France, 273)

D'autre part, Kinouchi accorde de l'importance au fait qu'en (17), le sujet est le pronom « on », qui n'a qu'une faible thématicité :

(17) On m'a volé mon portefeuille en allant à l'école. (51)

et dit que :

(18) « Pour que le sujet de la proposition régissante soit interprété comme contrôleur du gérondif, celui-là doit être un syntagme nominal avec une forte thématicité. Si la thématicité du syntagme nominal sujet est faible, un autre syntagme nominal avec une forte thématicité ou le thème du discours entier sera le contrôleur » (53, ma traduction).

Il y a pourtant une difficulté pour cette hypothèse : dans (19), le contrôleur du gérondif « en l'utilisant» est un sujet générique, glosable par « on », qui n'a, justement, qu'une faible thématicité selon Kinouchi :

(19) Mais le remonte-ski est un peu déséquilibré en l'utilisant seule. (ReichlerBéguelin §.3)

(19’) Mais le remonte-ski est un peu déséquilibré si on l'utilise seule.

Pour conclure le tour d'horizon des études antérieures, nous pourrions dire qu'en étendant le champ d'observation, leurs hypothèses butent souvent sur des contre-exemples et qu'il faudrait mener une enquête spécifique dans un corpus ciblé.

\section{Enquête dans le corpus}

Nous avons choisi 118 verbes élémentaires ${ }^{3}$ et relevé toutes les occurrences de ces verbes mis au gérondif (coréférentiel ou non-coréférentiel) dans le corpus « Corpatext $1.02 »{ }^{4}$. Au total, nous trouvons 35474 occurrences, dont 1482 (soit 4,2\%) sont non-coréférentielles.

\subsection{Types de procès des verbes au gérondif}

Nous avons rangé les occurrences selon cinq types de procès (répartis lexicalement comme dans la table 1 ci-dessous) exprimés par le verbe mis au gérondif. 
Table 1 : Classification des types de procès des verbes au gérondif

\begin{tabular}{|c|c|c|c|}
\hline $\begin{array}{c}\text { Type } \\
\text { de } \\
\text { procès }\end{array}$ & \begin{tabular}{||c|} 
Gérondifs \\
non- \\
coréférentiel \\
s \\
/ total
\end{tabular} & \begin{tabular}{||c|} 
Taux \\
d'occurrences \\
non- \\
coréféren- \\
tielles \\
\end{tabular} & $\begin{array}{c}\text { Détails } \\
\text { (occurrences non-coréférentielles / total) }\end{array}$ \\
\hline $\begin{array}{l}\text { psycho- } \\
\text { cognitif }\end{array}$ & $430 / 3375$ & $12,7 \%$ & \begin{tabular}{|l} 
en admettant $(116 / 143)$, en aimant $(3 / 95)$, en apercevant $(22 / 341)$, en \\
apprenant $(24 / 270)$, en choisissant $(1 / 51)$, en comparant $(9 / 109)$, en \\
comprenant $(1 / 35)$, en comptant $(34 / 132)$, en concevant $(0 / 8)$, en \\
connaissant $(7 / 35)$, en considérant $(23 / 129)$, en croyant $(3 / 137)$, en \\
étudiant $(7 / 75)$, en observant $(4 / 116)$, en oubliant $(0 / 32)$, en pensant \\
$(20 / 405)$, en reconnaissant $(8 / 184)$, en réfléchissant $(10 / 96)$, en sachant \\
$(3 / 62)$, en se demandant $(0 / 42)$, en se rappelant $(1 / 76)$, en songeant \\
$(14 / 322)$, en supposant $(116 / 271)$, en voulant $(4 / 209)$
\end{tabular} \\
\hline déplacement & $340 / 7644$ & $4,5 \%$ & $\begin{array}{l}\text { en allant }(16 / 352) \text {, en approchant }(35 / 155) \text {, en arrivant }(57 / 652) \text {, en } \\
\text { bougeant }(0 / 1) \text {, en courant }(2 / 506) \text {, en descendant }(8 / 248) \text {, en entrant } \\
(50 / 741) \text {, en marchant }(7 / 430) \text {, en montant }(9 / 207) \text {, en partant }(10 / 266) \text {, en } \\
\text { passant }(81 / 1575), \text { en rentrant }(9 / 389) \text {, en revenant }(11 / 399) \text {, en } \\
\text { s'approchant }(8 / 135) \text {, en se promenant }(2 / 134) \text {, en se rapprochant }(0 / 57) \text {, } \\
\text { en sortant }(23 / 710) \text {, en tombant }(0 / 205) \text {, en tournant }(2 / 235) \text {, en venant } \\
(2 / 225) \text {, en voyageant }(1 / 22)\end{array}$ \\
\hline perception & $158 / 4462$ & $3,5 \%$ & $\begin{array}{l}\text { en contemplant }(9 / 124) \text {, en écoutant }(20 / 275) \text {, en entendant }(22 / 412) \text {, en } \\
\text { regardant }(32 / 1361) \text {, en sentant }(1 / 89) \text {, en voyant }(74 / 2201)\end{array}$ \\
\hline activité & $518 / 19178$ & $2,7 \%$ & 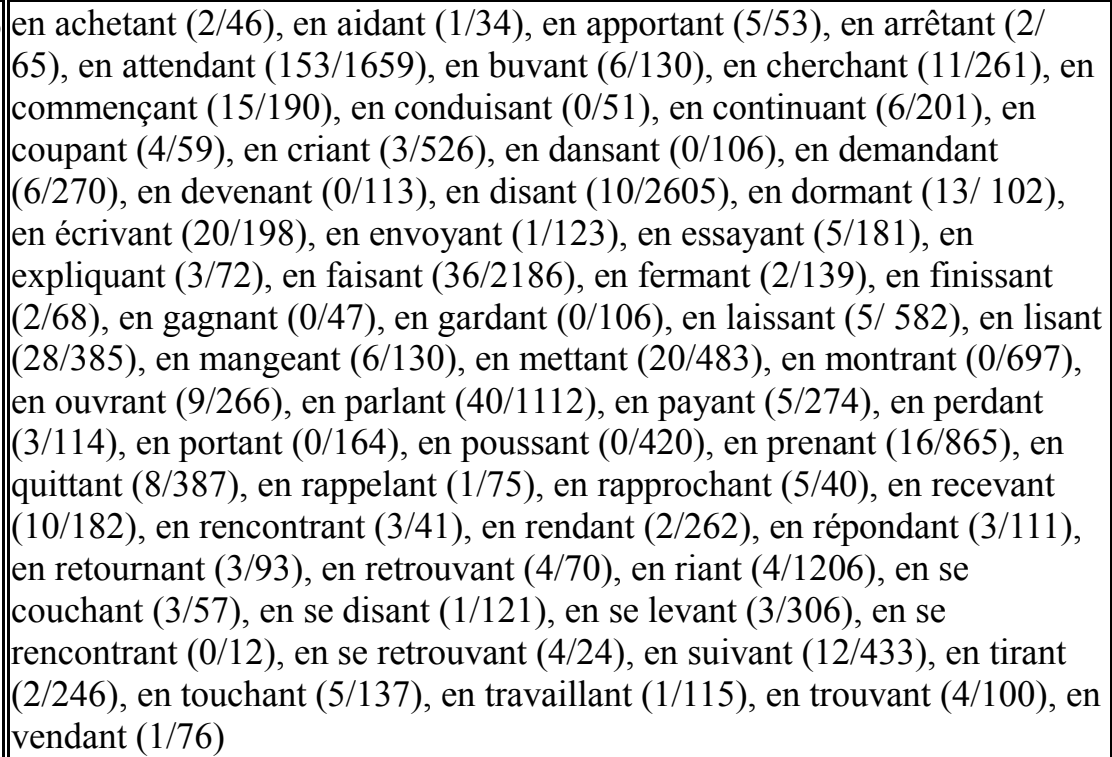 \\
\hline état & $36 / 816$ & $4,4 \%$ & $\begin{array}{l}\text { en ayant }(8 / 199) \text {, en étant }(0 / 93) \text {, en habitant }(0 / 1) \text {, en manquant }(1 / 9) \text {, en } \\
\text { restant }(4 / 151) \text {, en se trouvant }(1 / 41) \text {, en tenant }(21 / 251) \text {, en vivant }(1 / 71)\end{array}$ \\
\hline 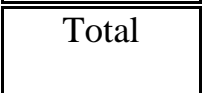 & $\begin{array}{l}1483 / \\
35474\end{array}$ & & \\
\hline
\end{tabular}

\subsubsection{Verbes psycho-cognitifs}

Parmi les cinq classes, c'est celle des verbes psycho-cognitifs qui contient le plus fréquemment des gérondifs non-coréférentiels $(12,7 \%)^{5}$.

Il y a beaucoup d'occurrences non-coréférentielles chez les verbes qui expriment les activités d'esprit relativement agentives et intentionnelles. Par exemple, en admettant (116 occurrences non-coréférentielles) et en supposant (116 occ.) ont un haut degré d'intentionnalité 
dans la mesure où ils expriment un acte de langage de haut niveau qu'est la supposition. Suivent, par ordre décroissant : en comptant (34 occ.), en apprenant (24 occ.), en considérant (23 occ.), en pensant (20 occ.), etc. En revanche, les verbes statiques comme en sachant (3 occ.), en croyant $(3$ occ.) et involontaires comme en oubliant (aucune occurrence noncoréférentielle) peuvent donner peu de gérondifs non-coréférentiels.

(20) Que de larmes me viennent en pensant à vous, à votre petite Marie, à sa mère qui m’a aimée ! (Eugénie de Guérin, Lettres)

(21) Malgré tout, en y réfléchissant, ces lettres passionnées ne répondaient nullement au physique de cette femme, car nulle n'était plus maîtresse des simagrées et plus calme. (JorisKarl Huysmans, Là-bas)

(22) En considérant bien, dit-il, ce qui vient de se passer entre la Floride et le Texas, il est évident que les mêmes difficultés se reproduiront entre les villes de l'état favorisé. (Jules Verne, De la Terre à la Lune)

(23) Lorsque, au repas du soir, cette descendance se trouvait réunie, ils étaient, en les comptant, sa femme et lui, trente et un à table. (Emile Zola, L'argent)

(24) Mais enfin, en supposant que toutes les difficultés soient résolues, tous les obstacles aplanis, en réunissant toutes les chances en votre faveur, en admettant que vous arriviez sain et sauf dans la Lune, comment reviendrez-vous ? (Jules Verne, De la Terre à la Lune)

\subsubsection{Verbes de déplacement}

Dans les cas non-coréférentiels, le syntagme gérondif représente le plus souvent un déplacement fictif ou un parcours fictif, qui est une sorte d'activité intellectuelle, proche de celles qu'indiquent les verbes psycho-cognitifs (§.2.1.1). Le contenu de la proposition régissante est souvent générique et atemporel, comme dans (25), (27).

Quand le syntagme gérondif représente un déplacement réel, il s'interprète souvent comme un repère temporel, et la proposition régissante comme un événement unique (semelfactif). Voir (26), (28).

(25) Autour de lui [=le Soleil] gravitent huit planètes, sorties de ses entrailles mêmes aux premiers temps de la Création. Ce sont, en allant du plus proche de ces astres au plus éloigné, Mercure, Vénus, la Terre, Mars, Jupiter, Saturne, Uranus et Neptune. (Jules Verne, De la Terre à la Lune)

(26) Elle [=Béatrix Cenci] avait la bouche petite, les cheveux blonds et naturellement bouclés. En allant à la mort ces cheveux blonds et bouclés lui retombaient sur les yeux ce qui donnait une certaine grâce et portait à la compassion. (Stendhal, Les Cenci) 
(27) Enfin, en descendant vers notre âge, commencent ces voyages modernes où la civilisation laisse briller toutes ses ressources, la science tous ses moyens. (François René de Chateaubriand, Voyage en Amérique)

(28) En descendant de la carriole, le cœur lui battait bien fort, et la maison du beaupère-chaumière misérable et croulante-lui apparut plus splendide que tous les palais des contes de fées. (Octave Mirbeau, Le concombre fugitif)

Parallèlement au cas des verbes psycho-cognitifs, les occurrences non-coréférentielles sont nombreuses chez les verbes qui représentent un déplacement volontaire et intentionnel. Les verbes exprimant un déplacement involontaire connaissent peu d'occurrences noncoréférentielles (par exemple, en tombant n'en connaît aucune).

Les verbes qui représentent une manière de déplacement connaissent peu d'occurrences non-coréférentielles (par exemple, en tournant : aucune, en courant : 2 occurrences). La raison en est qu'une écrasante majorité des gérondifs indiquant une " manière » sont coréférentiels (cf. (29) et $\S .1 .2)$.

(29) Il sort en courant, après avoir envoyé des baisers à Yesouf. (Louis Fréchette, Véronica)

\subsubsection{Verbes de perception}

C'est en voyant qui donne le plus d'occurrences non-coréférentielles (74 occ.), et en sentant, le moins ( 1 occ.). Il n'y a pas de tendance particulière entre les verbes de vue et d'ouïe.

La tendance vers la haute agentivité ou intentionnalité, que nous avons constatée pour d'autres catégories de verbes, n'est pas toujours attestée ici. Le taux d'occurrences noncoréférentielles d'en entendant est de 5,6\%, celui d'en écoutant de 7,3\% ; mais en voyant de $3,4 \%$, en regardant de 2,4\%. Cela tient peut-être au fait que les verbes de perception ne sont pas assez nombreux pour pouvoir reproduire la tendance générale.

La proposition régissante représente soit la réaction du sujet percepteur à la perception, soit l'impression qu'il éprouve de la perception.

(30) Tout en écoutant son mari qui parlait d'un air grave, l' ail de Mme de Rênal suivait avec inquiétude les mouvements de trois petits garçons. (Stendhal, Le rouge et le noir) ${ }^{6}$

(31) D'autres fois il semble qu'il y a des révoltes dans les sens de votre corps, même en voyant, en touchant, en entendant ${ }^{7}$ et en sentant les choses saintes; quand vous vous en approchez, on dirait que tout apporte à vos sens un trouble honteux et corrupteur. (Sainte Catherine de Sienne, Traité de la providence)

(32) Souvent, en la regardant, il lui semblait que son âme, s'échappant vers elle, se 
répandait comme une onde sur le contour de sa tête, et descendait entraînée dans la blancheur de sa poitrine. (Gustave Flaubert, Madame Bovary)

(33) Mon espoir disparut, en voyant tes différentes liaisons avec les Donis, les Grillo, les Borghèse, et je me désespérai bien plus encore, quand je sus que tu avais retrouvé Clairwil... (Marquis de Sade, Histoire de Juliette)

\subsubsection{Verbes d'activité}

Parmi les verbes d'activité, ceux qui expriment les actions accompagnées de fonctionnements mentaux, ceux qui sont donc proches de la catégorie des verbes psychocognitifs, à savoir en attendant (116 occurrences non-coréférentielles), en parlant (40 occ.), en lisant (28 occ.), en écrivant (20 occ.) etc., donnent beaucoup de gérondifs non-coréférentiels. Par contre, ceux qui sont proches des verbes d'état connaissent peu d'occurrences noncoréférentielles (par exemple en gardant, en portant qui n'en connaissent aucune).

(34) Ma grande découverte en lisant cet article est qu'il y avait largement plein de trucs intéressants qui se passaient là où je n'étais pas. (Pierre Ernoult, Rovaniemi)

(35) En écrivant ces lignes vengeresses, le rouge me monte au front ; car il y a malheureusement des auteurs de SF qui sont les suiveurs de Boileau (avant qu'il ne connût Narcejac). (Jacques Sadoul, Mothers of Invention)

(36) Laisse-moi donc continuer, la peine se dissipe en parlant ; [...] (Louis Bouilhet, Melaenis, conte romain)

Il y a beaucoup d'expressions figées qui font de verbes d'activité des locutions verbales psycho-cognitives. Par exemple, parmi les 36 occurrences d'en faisant, 19 forment ce genre de locutions : faire abstraction, faire l'économie, faire exception, etc.

(37) Et cette question - l'avènement de la pensée anticipatrice - ne pourra être élucidée en faisant l'économie de la pensée du temps : l'événement est ce qui advient à l'être lorsqu'il perd son indétermination première pour se différencier comme étant. (Patrice Deramaix, Logos et totalité)

\subsubsection{Verbes d'état}

Avec ce groupe, il y a le moins d'occurrences non-coréférentielles par verbe. Par exemple, en étant, en se trouvant, en vivant, etc. n'en connaissent aucune ${ }^{8}$. Parmi les occurrences non-coréférentielles, on trouve beaucoup de locutions de sens psycho-cognitif comme en ayant soin, en ayant égard, en tenant compte, etc. C'est le passage à ces locutions qui « sauve » les verbes d'état.

(38) Rien ne doit donc empêcher la France d'entrer dans des négociations en ayant soin 
de les rapprocher le plus possible de l'esprit dans lequel cette Note est rédigée. (Chateaubriand, Mémoires d'outre-tombe)

(39) Sur terre, les temps de la chasse sont réglés ; ceux de la pêche doivent l'être également, en ayant égard aux saisons où se reproduit chaque espèce. (Jules Michelet, La mer)

(40) Le siège d'autres organismes et services créés ou à créer sera décidé d'un commun accord par les représentants des gouvernements des États membres lors d'un prochain Conseil européen, en tenant compte des avantages des dispositions ci-dessus pour les États membres intéressés et en donnant une priorité appropriée aux États membres qui, à l'heure actuelle, n'abritent pas le siège d'une institution des Communautés. (Décision prise du commun accord des représentants des gouvernements des États membres relative à la fixation des sièges des institutions et de certains organismes et services des Communautés européennes (JOC $341 \mathrm{du}$ 23/12/1992))

\subsubsection{Conclusion de la section 2.1.}

On peut constater que les verbes psycho-cognitifs sont prototypiques pour le gérondif non-coréférentiel. Les verbes des autres catégories tendent à être facilement employés dans le gérondif non-coréférentiel lorsqu'ils revêtent un caractère proche des verbes psycho-cognitifs.

Les verbes avec une haute agentivité ou intentionnalité sont préférés dans le gérondif non-coréférentiel. Ceux qui expriment le procès statique ou involontaire ne le sont pas.

\subsection{Caractéristiques de la proposition régissante}

Quant aux caractéristiques de la proposition régissante qui héberge le gérondif noncoréférentiel, nous les abordons sous deux angles : traits syntactico-sémantiques de la proposition régissante et aspects lexicaux du verbe ${ }^{9}$. 
Table 2 : Caractéristiques de la proposition régissante des gérondifs non-coréférentiels

\begin{tabular}{|c|c|c|c|c|c|c|c|c|c|c|c|}
\hline \multirow[b]{2}{*}{ Type de procès } & \multicolumn{8}{|c|}{$\begin{array}{l}\text { (i) traits syntactico-sémantiques de la proposition régissante } \\
\text { (cumul de plusieurs conditions admis, sauf }(*) \text { ) }\end{array}$} & \multicolumn{3}{|c|}{$\begin{array}{l}\text { (ii) aspects lexicaux } \\
\text { du verbe }\end{array}$} \\
\hline & 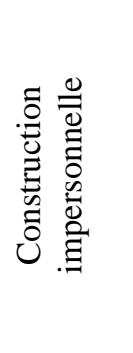 & 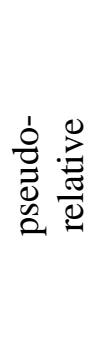 & 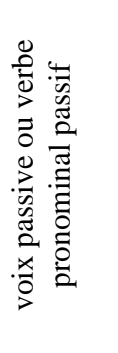 & 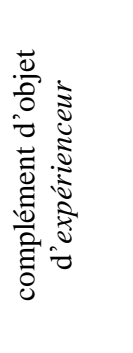 & 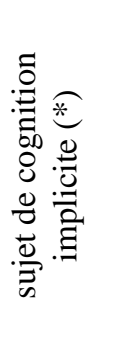 & 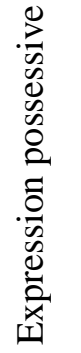 & 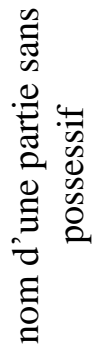 & 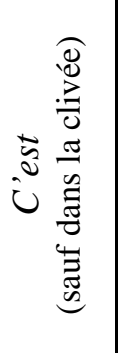 & 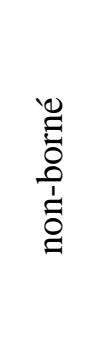 & $\stackrel{\mathscr{\Xi}}{0}$ & 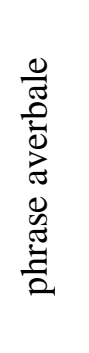 \\
\hline "psycho-cognitif & 83 & 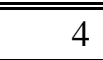 & 14 & 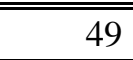 & 222 & 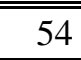 & 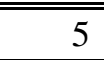 & 9 & ב331 & 81 & 18 \\
\hline déplacement & 48 & 9 & 14 & 77 & 139 & 69 & 9 & 6 & 254 & 75 & 13 \\
\hline perception & 22 & 0 & 4 & 40 & 32 & 62 & 7 & 2 & 106 & 51 & 1 \\
\hline activité & 109 & 11 & 33 & 112 & 164 & 103 & 6 & 14 & 386 & 93 & 36 \\
\hline état & 7 & 0 & 5 & 5 & 16 & 2 & 1 & 0 & 29 & 7 & 2 \\
\hline total & 269 & 24 & 70 & 283 & 573 & 290 & 28 & 31 & 1106 & 307 & 70 \\
\hline
\end{tabular}

(i) Traits syntactico-sémantiques de la proposition régissante. La condition « sujet

de cognition implicite » l'emporte largement. En fait, nous n'avons reconnu cette condition que si aucune autre condition n'est trouvée en même temps. Sinon, elle recouvrirait la presque totalité des gérondifs non-coréférentiels sauf ceux qui sont en cooccurrence avec le complément d'objet d'expérienceur. Elle est donc la condition la plus importante et prototypique du gérondif non-coréférentiel.

(ii) Aspects lexicaux du verbe. L'aspect non-borné, dont le prototype est l'aspect statique, occupe la majorité (74,6\%) des occurrences non-coréférentielles.

De (i) et (ii), nous pourrions dire que la prédication d'une situation avec un point de vue intérieur à celle-ci est prototypique pour l'énonciation du gérondif non-coréférentiel.

Voyons ci-dessous les traits syntactico-sémantiques cas par cas.

\subsubsection{Construction impersonnelle}

La construction impersonnelle décrit directement l'état de choses, avec peu de traces du sujet. Le sujet de cognition n'est souvent pas explicité, comme dans (41), mais on peut parfois l'entrevoir sous la forme du C.O.I. d'expérienceur, comme dans (42).

(41) Or, à deux cent quarante milles par douze heures, ce qui n'approche pas de la vitesse de nos chemins de fer, en voyageant jour et nuit, il suffirait de sept jours pour traverser l'Afrique. (Jules Verne, Cinq semaines en ballon)

(42) [...] je vous avoue que je ne me suis pas trop informé de quelle manière il plaît à Dieu d'exercer sa justice, quoique, à vous dire vrai, il me semble, en réfléchissant sur ce qui se passe dans le monde, que s'il punit dès cette vie, au moins il ne se presse pas. (Joseph de 
Maistre, Les soirées de Saint-Pétersbourg)

\subsubsection{Pseudo-clivée}

La pseudo-clivée en ce qui / que..., c'est... sert à expliciter la structure informationnelle en divisant les parties thématique et rhématique dans la phrase. Dans une écrasante majorité des cas, le sujet formel ce que.../qui... n'est pas compatible avec celui du gérondif, qui, lui, correspond à un sujet de cognition concret ${ }^{10}$. Le cadre formel de la pseudo-clivée fonctionne au niveau métacognitif, niveau plus haut que la structure argumentale du verbe.

(43) Ce qui, en entrant, frappait d'abord le regard, c'était entre deux larges fenêtres une haute statue de la Liberté. (Victor Hugo, Quatre-vingt-treize)

(44) Ce que je vis, en ouvrant la porte, me terrifia. (Alphonse Daudet, Le Petit Chose)

\subsubsection{Voix passive ou verbe pronominal passif}

La voix passive et le verbe pronominal passif dans la proposition régissante causent la non-coréférence quand le complément d'agent devient le sujet du gérondif.

(45) Or on a vu que, sur ce point aussi, Leibniz s'accrochait avec Descartes : puisque le second critère de la substance chez Descartes c'était-vous vous rappelez-la distinction réelle : que deux choses soient conçues sans que l'une soit pensée en faisant intervenir des éléments de l'autre. (Deleuze, Leibniz)

(46) L'extrait gras du haschisch, tel que le préparent les Arabes, s'obtient en faisant bouillir les sommités de la plante fraîche dans du beurre avec un peu d'eau. (Charles Baudelaire, Les paradis artificiels)

Ces constructions ont la vertu de repousser l'agent à l'arrière-plan et de faire une phrase avec moins de transitivité, une phrase du «type devenir » d'Yoshihiko Ikegami (1991), qui correspond à un trait du mode de cognition « mode I» que nous mentionnerons plus loin.

\subsubsection{Complément d'objet d'expérienceur ${ }^{11}$}

Le complément d'objet d'expérienceur, soit complément direct (47), soit indirect (48), est un argument que revendique le verbe psychologique (Ruwet Théorie, « Verbes »). En comparaison aux cas où le sujet du gérondif devient l'expérienceur, qui explicitent « objectivement» la relation entre le sujet de cognition et le contenu observé, ceux qui prennent un argument expérienceur décrivent des faits ou des états psychologiques dans lesquels l'expérienceur « plonge », pour ainsi dire.

(47) La première personne qui le frappa en entrant fut Aramis, installé près d'un fauteuil à roulettes, fort large, recouvert d'un dais en tapisserie, sous lequel s'agitait, enveloppée dans une couverture de brocart, une petite figure assez jeune, assez rieuse, mais 
parfois pâlissante, sans que ses yeux cessassent néanmoins d'exprimer un sentiment vif, spirituel ou gracieux. (Alexandre Dumas, Vingt ans après)

(48) [...] en marchant, la lune, qu'elle avait à sa gauche et sur le fil de l'horizon, $\underline{\text { lui }}$ semblait marcher du même pas qu'elle, et lui faisait l'effet d'une tête de mort qui l'aurait obstinément accompagnée. (Jules Barbey d'Aurevilly, Une histoire sans nom)

\subsubsection{Sujet de cognition implicite}

Cette condition, la plus fréquente même si on rejette tous les cumuls avec les autres conditions, peut être considérée comme prototypique pour le gérondif non-coréférentiel. En effet, dans les exemples que nous avons rangés sous d'autres rubriques aussi, cette condition est omniprésente. Par exemple, (49) dispose de la condition de la construction impersonnelle, (50) celle du verbe pronominal passif, outre l'existence du sujet de cognition implicite. Le contenu de la proposition régissante est le contenu de cognition par le sujet implicite.

(49) Il y eut bien encore, en commençant, quelque débat entre nous sur la manière dont j'avais besoin moi aussi, d'être aimé. (Sainte-Beuve, Volupté)

(50) Elle [=la migration] est essentielle aussi à un développement futur de ces communautés, développement qui ne se peut se concevoir - en raison même de la politique économique imposée par le FMI - en faisant abstraction de l'économie de marché et des dépendances technico-économiques qu'elle entraîne. (Patrice Deramaix, L'exil, l'épreuve et le défi)

L’omniprésence de cette condition explique également la haute fréquence, que nous avons vue dans §.2.1.1, des verbes psycho-cognitifs mis en gérondif non-coréférentiel, puisque ce sont ces derniers qui renvoient la cognition par le sujet implicite.

\subsubsection{Expressions possessives (adjectif possessif, préposition de, pronom relatif dont, etc.)}

Les expressions possessives dans la proposition régissante sont métonymiques dans la mesure où elles servent à parler de l'action du possesseur à travers l'action ou l'état de sa possession. C'est la différence entre possesseur et possession qui provoque la non-coréférence.

(51) Alors, en m'approchant pour l'embrasser, ma main rencontra sa main, sa chère main toute moite des sueurs de l'agonie. (Alphonse Daudet, Le Petit Chose)

(52) Vers onze heures une cavalcade assez nombreuse entra dans Pietranera; c'étaient le colonel, sa fille, leurs domestiques et leur guide. En les recevant, le premier mot de Colomba fut : «Avez-vous vu mon frère ? »(Prosper Mérimée, Colomba)

Ce sont les noms renvoyant aux organes de sensation ou de pensée ou aux sentiments vécus (ses yeux, son caur, sa tête; son émotion, son sentiment, son attention, etc.) qui occupent 
souvent la position de possession. Étant donné le sens de ces noms, ces cas ressemblent plus ou moins aux cas de cooccurrence avec les compléments d'expérienceur (\$.2.2.4) :

(53) Et mon cœur se fondait en délices en pensant aux voluptés que donnerait ce baiser. (Gustave Flaubert, Mémoires d'un fou)

(54) Mon étonnement s'accrut en voyant que cette immense énumération se composait seulement des personnes qui se trouvaient dans la salle et dont $\mathrm{j}$ 'avais vu les images se diviser et se combiner en mille aspects fugitifs. (Gérard de Nerval, Aurélia)

Qui plus est, ces substantifs peuvent occuper la place de ces derniers dans la phrase :

(55) En entrant dans la salle à manger, le spectacle le plus inattendu frappa mes regards. (Anatole France, La cravate)

\subsubsection{Nom d'une partie sans possessif}

Les expressions du type « article défini + nom d'une partie du corps » fonctionnent d'une façon très semblable aux possessifs que nous avons vus dans la section précédente.

(56) En l'écoutant, toutes les têtes étaient penchées sur les poitrines, toutes les oreilles étaient tendues vers cette voix qui planait, comme la foudre, sous ces voûtes émues. (Jules Barbey d'Aurevilly, Une histoire sans nom)

(57) Le caur lui battait très fort, en entrant dans le petit hôtel de la rue Legendre, cette maison cossue où elle avait grandi et où elle croyait ne plus trouver que des étrangers, tellement l'air lui semblait, autre, glacial. (Emile Zola, L'argent)

\subsubsection{C'est... ou ses variantes (sauf dans la clivée en c'est... que)}

\subsubsection{Gérondif dans la position thématique}

Lorsque le gérondif paraît dans la position thématique, on peut l'interpréter comme une condition pour le contenu de la proposition régissante.

(58) En prenant une saison favorable pour passer d'ici aux Indes, c'est un voyage de six semaines au plus, et d'autant de temps pour en revenir. (Bernardin de Saint-Pierre, Paul et Virginie)

(59) L'échange n'a duré qu'une minute, sur un sujet banal. Sur le moment, je n'y ai prêté aucune attention, mais en y réfléchissant, c’est inhabituel. (Pierre-Jean Bascuñanam, Demain)

\subsubsection{Gérondif dans la position d'attribut}

Quand le gérondif vient dans la position d'attribut, nous le classifions comme noncoréférentiel, car le sujet syntaxique de la proposition ce ne peut jamais être le sujet du gérondif. Mais d'un point de vue informationnel, on peut considérer qu'ici c'est sert simplement à 
focaliser le complément circonstanciel (on peut paraphraser (60) par une phrase clivée (60’)). Nous devrions exclure ces cas d'une véritable non-coréférence.

(60) On protège tant qu'on peut la retraite ; on se bat vigoureusement en reculant, mais c'est en perdant encore beaucoup de monde ; et l'on a toujours été surpris que les français aient pu regagner leur camp. (Baudry des Lozières, Voyage à la Louisiane et sur le continent de l'Amérique septentrionale)

$\approx\left(60^{\prime}\right)$ C'est en perdant encore beaucoup de monde qu'on se bat vigoureusement en reculant.

\subsubsection{Conclusion de la section 2.2.}

La condition discutée dans la section 2.2.5, à savoir celle du sujet de cognition implicite, est si largement observée que nous la considérons comme prototypique. Les autres conditions entrent en harmonie avec celle-ci.

\section{Le gérondif non-coréférentiel et les modes de cognition}

\subsection{La cohérence du gérondif non-coréférentiel}

En récapitulant les conclusions de $\S .2 .1$ et de $\S .2 .2$, nous pourrions dire que c'est le sujet de cognition sous-jacent à la proposition régissante qui est le sujet implicite du gérondif non-coréférentiel. La cohérence de la phrase, rompue en apparence par le fait de la non-coréférence, est sauvée par l'invariance de ce sujet de cognition.

\subsection{Le " mode D " et le " mode I"}

En linguistique cognitive, la distinction de Ronald Langacker (1985) entre « configuration optimale (ou canonique) de vue » et « configuration égocentrique de vue » est bien connue : « la configuration optimale de vue, définie comme celle qui maximalise l'asymétrie entre voyeur-vu » (121, ma traduction) et « la configuration égocentrique de vue qui correspond aux cas où l'observateur est spécialement concerné par le « soi » et fonctionne donc à la fois comme le conceptualisateur et comme l'objet de la conceptualisation » (123, ma traduction).

Au Japon, Yoshihisa Nakamura critique l'étroitesse de la conception de Langacker qui ne prend en compte que ce qui est réductible à la relation voyeur-vu ${ }^{12}$. Et il propose, comme alternative, de distinguer un «mode de cognition déplacé » (abrégé en « mode $\mathrm{D} »)$ et un «mode de cognition par interaction » (« mode $\mathrm{I} »){ }^{13}$. Avec le mode $\mathrm{D}$, le sujet reconnaît l'objet en se situant (fictivement) en dehors du champ de cognition. Avec le mode I, l'acte de cognition interagit avec l'objet au sein du monde vécu. (358-365) 
Sur la base des comparaisons de vingtaine de traits morphologiques, syntaxiques, sémantiques et stylistiques de chaque langue, Nakamura et Yakusata Haruki affirment que l'anglais est une langue avec une forte tendance vers le mode $\mathrm{D}$, le japonais au contraire vers le mode I, le français intermédiaire. Voici des exemples qui illustrent ce contraste général :

(61) Kunizakaino nagai tunnelwo nukeruto, yukugunide atta. (Yasunari Kawabata, Pays de neige, incipit)

(Littéralement : «Au sortir du long tunnel de la frontière, c'était le pays de neige. »)

(62) The train came out of the long tunnel into the snow country. (tr. Edward Seidensticker)

(63) Un long tunnel entre les deux régions, et voici qu'on était dans le pays de neige (tr. Bunkichi Fujimori et Armel Guerne)

La version originale (61) décrit la situation telle qu'on voit par la fenêtre du train avec le point de vue qui pénètre dans la réalité, ce qui correspond au mode I. La traduction anglaise (62) met le sujet the train pour garder le point de vue extérieur et objectif, extrêmement fidèle au mode D. La traduction française (63) fait de la première moitié de la phrase un seul syntagme nominal, et commence la deuxième moitié par un déictique voici, ce qui donne le sentiment de la réalité vécue, beaucoup plus proche de l'original que la version anglaise.

\subsection{Le gérondif non-coréférentiel comme une expression selon le mode I}

Notre conclusion est que le gérondif non-coréférentiel est une façon d'expression qui reflète le mode $I$.

La condition prototypique du gérondif non-coréférentiel, à savoir l'existence du sujet de cognition implicite, est un phénomène du mode I par excellence. Ce sujet de cognition laisse parfois sa trace dans les expressions comme le complément expérienceur ou les possessifs, mais même dans ces cas-là, il s'agit plutôt d'un phénomène du mode I, si on les compare avec les cas de gérondif coréférentiel, qui sont, eux, plutôt au mode D.

Prenons le cas d'en supposant : dans les cas de coréférence, le sujet de cognition est actualisé comme le sujet syntaxique de la proposition régissante.

(64) En supposant qu'après avoir mangé le cuir ils mangent la semelle, je ne vois pas trop ce qui leur restera après, à moins de se manger les uns les autres. (Alexandre Dumas, Les trois mousquetaires)

(65) Marguerite connaissait si bien son père qu'elle devina le motif de cette tendresse, en supposant qu'il pouvait avoir en ville quelques dettes desquelles il voulait s'acquitter avant son départ. (Balzac, La recherche de l'absolu)

Par contre, dans les cas de non-coréférence, le sujet de cognition n'a pas nettement de 
place dans la phrase :

(66) Mais d'abord, en supposant que le jeune comte n'ait pas eu le droit de tirer sur du Croisier, il n'y aurait pas imitation de signature. (H. de Balzac, Le cabinet des antiques)

(67) M. Leconte de Lisle est la première et l'unique exception que j'ai rencontrée. En supposant qu'on en puisse trouver d'autres, il restera, à coup sûr, la plus étonnante et la plus vigoureuse. (Baudelaire, Curiosités esthétiques)

Un autre fait à l'appui de notre hypothèse : en traduisant littéralement le gérondif noncoréférentiel du français en japonais, langue au mode I par excellence, on obtient des phrases très naturelles, " très japonaises " :

(68) $[=(1)]$ L'appétit vient en mangeant, la soif s'en va en buvant.

(68') Shokuyokuwa tabeteiruto kuruga, kawakiwa nondeiruto saru.

(69) $[=(2)]$ En le retrouvant à la sortie dans les jardins, il était avec un homme, il s'était fait alpaguer par un pédophile.

(69') Teien'eno deguchide kareto mata auto, karewa hitorino otokoto ita. Shounenaishani iiyorarete itanoda.

\section{Conclusion}

Selon Jean-Claude Chevalier et al :

(70) «L'exigence d'un sujet identique pour la principale et le groupe subordonné ne date que du XVIII ${ }^{\text {ème }}$ siècle. On usait auparavant d'une bien plus grande liberté : [...] Mes crimes, en vivant, me la pourraient ôter. (Corneille) » (126-127)

L'introduction de la règle de coréférence était, nous semble-t-il, un mouvement rationaliste qui exigeait que l'on décrive la situation par un point de vue extérieur et objectif, par le « mode D ». Mais malgré les reproches des grammaires normatives, on n'a pas cessé d'employer le gérondif non-coréférentiel, qui répond bien au besoin d'exprimer la situation avec un point de vue intérieur à celle-ci, autrement dit, avec le «mode I ». Nous croyons avoir pu montrer que la «transgression » commise par le gérondif non-coréférentiel a sa propre logique, sa propre raison d'être.

\section{Bibliographie}

Chevalier, Jean-Claude et al. Grammaire de français contemporain. $2^{\mathrm{e}}$ éd. Paris : Larousse, 1988.

Grevisse, Maurice et André Goose. Le bon usage. 13e éd. Louvain-la-Neuve : Duculot, 1993. Halmøy, Odile. Le gérondif en français. Paris : Ophrys, 2003. 
Haruki, Yasutaka. « Francegono ninchi-mode-ni tsuite ». Gengoni okeru jikuuwo megutte 9 (2011) : 61-70.

Haspelmath, Martin. « The Converb as a Cross-Linguistically Valid Category ». Converbs in Cross-linguistic Perspective. Dir. Martin Haspelmath et Ekkehard König. Berlin : Mouton de Gruyter, 1995. 1-55.

Hayase, Naoko. « The Cognitive Motivation for the Use of Dangling Participles in English ». Motivation in Grammar and the Lexicon. Dir. Klaus-Uwe Panther et Günter Radden. John Benjamins, 2011. 89-106.

Ikegami, Yoshihiko. " 'DO-language' and 'BECOME-language' : Two Contrasting Types of Linguistic Representation ». The Empire of Signs : Semiotic Essays on Japanese Culture. Dir. Ikegami, Yoshihiko. John Benjamins, 1991. 285-326.

Kinouchi, Yoshiyuki. Francegono tougoron kenkyuu. Tokyo : Keisoushoboo, 2005.

Kleiber, Georges. « Gérondif et manière ». Langue française 171 (2011) : 117-134.

Langacker, Ronald. " Observations and Speculations on Subjectivity ». Iconicity in Syntax. Dir. John Haiman. John Benjamins, 1985. 109-150.

Legendre, Géraldine. « Inversion with certain French Experiencer Verbs ». Languages $65: 4$ (1989) : 752-782.

Nakamura, Yoshihisa. «Ninchi-mode-no shatei ». Uchito Sotono Gengogaku. Éds. Tsubomoto, Atsushi et al. Tokyo : Kaitakusha, 2009. 353-393.

Reichler-Béguelin, Marie-José. « Les problèmes d'emploi du gérondif et des participiales en français contemporain ». Le français langue étrangère à l'université : théorie et pratique. Dir. Krystyna Zaleska et Annick Cataldi. Uniwersytet Warszawski : Instytut Romanistyki, 1995. 243-260.

Ruwet, Nicolas. Théorie syntaxique et syntaxe du français. Paris : Seuil, 1972.

---. « Les verbes dits psychologiques : trois théories et quelques questions ». Recherches linguistiques de Vincennes 22 (1993) : 95-124.

\footnotetext{
NOTES

${ }^{1}$ Nous remercions, entre autres, Naoko Hayase, Naoaki Wada et Baptiste Puyo pour leur aide amicale en vue de cet article. Nous avons bénéficié du soutien financier de la Subvention pour les Recherches Scientifiques (C) 24520530 et (C) 25370422 du Ministère de l'Éducation et des Sciences du Japon.

${ }^{2}$ La frontière entre les emplois marquant la concomitance et la manière n'est pas évidente. Voir à ce propos Kleiber.

${ }^{3}$ Voir la rubrique « détails » de la table 1 dans la section 2.1.

4 Un corpus de 2700 textes et de 37 millions de mots, téléchargeable à [http://www.lexique.org/public/lisezmoi.corpatext.htm]
} 


\footnotetext{
${ }^{5}$ Nous avons effectué la classification des verbes mécaniquement en attribuant à chacun une classe lexicalement prototypique, ce qui rend inévitable quelque décalage par rapport à la véritable typologie de toutes les occurrences. Pourtant, la tendance vers les verbes psycho-cognitifs serait encore plus accentuée si nous cherchions davantage d'exactitude dans la classification, puisqu'on a recours souvent aux verbes d'action comme prendre ou aux verbes d'état comme tenir pour exprimer le procès psycho-cognitif, à travers des expressions telles que prendre qqch. pour qqch., prendre conscience, tenir compte, etc., mais l'inverse n'est pas le cas.

${ }^{6}$ Contre-exemple à l'affirmation que les gérondifs précédés de tout seraient toujours coréférentiels.

${ }^{7}$ Le verbe entendre est polysémique, mais curieusement, pour le gérondif en entendant, seul le sens d'ouïe est attesté, à la différence du participe présent simple entendant : «Les tragédies et tragi-comédies seraient du pur hébreu pour ces rustiques ignorants de l'histoire et de la fable, et n'entendant pas même le beau langage français. » (Théophile Gautier, Le Capitaine Fracasse). Pour le moment, nous devons reconnaître que nous ne sommes pas en mesure d'expliquer ce fait.

${ }^{8}$ Rien d'étonnant à ce phénomène, si on se souvient de la tendance, constatée dans les sections précédentes, vers la haute agentivité : les verbes d'état sont au contraire ceux les moins agentifs.

${ }^{9}$ Nous avons classifié les occurrences également selon les temps et les modes du verbe de la proposition régissante, mais nous n'arrivons pas à trouver de tendance particulière.

${ }^{10}$ Voici un des rares exemples où le sujet implicite du gérondif correspond exactement à l'antécédent formel de се quе... : «Ce que Bergotte me dit au sujet de Cottard me frappa, tout en étant contraire à tout ce que je croyais » (Proust, À l'ombre des jeunes filles en fleurs).

${ }^{11}$ Celui qui éprouve le sentiment ou la perception. Ce terme est un calque d' « experiencer» en anglais, auquel on préfère parfois le terme, plus français, d' « expérient ».

${ }^{12}$ En outre, nous pourrions formuler une autre critique contre Langacker (1985), selon laquelle rien n'est plus « égocentrique » que de prétendre « optimale » ou «canonique » la vision prototypique dans sa propre langue ou culture!

${ }^{13}$ Concernant les modes I et D de Nakamura, on trouvera une excellente présentation par Werner Abraham dans la préface de la traduction anglaise de la Sprachtheorie de Karl Bühler publiée en 2011 chez Benjamins.
} 